\title{
Congruences of Multipartition Functions Modulo Powers of Primes
}

\author{
William Y.C. Chen ${ }^{1}$, Daniel K. Du², Qing-Hu Hou ${ }^{3}$ and Lisa H.Sun ${ }^{4}$ \\ Center for Combinatorics, LPMC-TJKLC \\ Nankai University, Tianjin 300071, P. R. China \\ E-mail: ${ }^{1}$ chen@nankai.edu.cn, ${ }^{2}$ dukang@mail.nankai.edu.cn, \\ ${ }^{3}$ hou@nankai.edu.cn, ${ }^{4}$ sunhui@nankai.edu.cn
}

\begin{abstract}
Let $p_{r}(n)$ denote the number of $r$-component multipartitions of $n$, and let $S_{\gamma, \lambda}$ be the space spanned by $\eta(24 z)^{\gamma} \phi(24 z)$, where $\eta(z)$ is the Dedekind's eta function and $\phi(z)$ is a holomorphic modular form in $M_{\lambda}\left(\mathrm{SL}_{2}(\mathbb{Z})\right)$. In this paper, we show that the generating function of $p_{r}\left(\frac{m^{k} n+r}{24}\right)$ with respect to $n$ is congruent to a function in the space $S_{\gamma, \lambda}$ modulo $m^{k}$. As special cases, this relation leads to many well known congruences including the Ramanujan congruences of $p(n)$ modulo 5,7,11 and Gandhi's congruences of $p_{2}(n)$ modulo 5 and $p_{8}(n)$ modulo 11. Furthermore, using the invariance property of $S_{\gamma, \lambda}$ under the Hecke operator $T_{\ell^{2}}$, we obtain two classes of congruences pertaining to the $m^{k}$-adic property of $p_{r}(n)$.
\end{abstract}

AMS Classification. 05A17, 11F33, 11P83

Keywords. modular form, partition, multipartition, Ramanujan-type congruence

\section{Introduction}

The objective of this paper is to use the theory of modular forms to derive certain congruences of multipartitions modulo powers of primes.

Recall that an ordinary partition $\lambda$ of a nonnegative integer $n$ is a nonincreasing sequence of positive integers whose sum is $n$, where $n$ is called the weight of $\lambda$. The partition function $p(n)$ is defined to be the number of partitions of $n$. A multipartition of $n$ with $r$ components, as called by Andrews [2], also referred to as an $r$-colored partition, see, for example [7,9], is an $r$-tuple $\lambda=\left(\lambda^{(1)}, \ldots, \lambda^{(r)}\right)$ of partitions whose weights sum to $n$. The number of $r$-component multipartitions of $n$ is denoted by $p_{r}(n)$.

Multipartitions arise in combinatorics, representation theory and physics. As pointed out by Fayers [10], the representations of the Ariki-Koike algebra are naturally indexed by multiparititions. Bouwknegt [6] showed that the Durfee square formulas of multipartitions are useful in deriving expressions for the characters of modules of affine Lie algebras in terms of the universal chiral partition functions. 
For the partition function $p(n)$, Ramanujan [23 26] proved that

$$
p(A n+B) \equiv 0 \quad(\bmod M),
$$

for all nonnegative integers $n$ and for $(A, B, M)=(5,4,5),(7,5,7)$ and $(11,6,11)$. In general, congruences of form (1.1) are called Ramanujan-type congruences. For $m=5$ and 7, Watson [29] proved that

$$
p\left(m^{k} n+\beta_{m, k}\right) \equiv 0 \quad\left(\bmod m^{k}\right),
$$

where $k \geq 1$ and $\beta_{m, k} \equiv 1 / 24\left(\bmod m^{k}\right)$. Atkin [3] showed that (1.2) is also valid for $m=11$. When $M$ is not a power of 5,7 or 11, Atkin and O'Brien [5] discovered the following congruence

$$
p\left(11^{3} \cdot 13 n+237\right) \equiv 0 \quad(\bmod 13) .
$$

Using the theory of modular forms, Ono 21] proved that for any prime $m \geq 5$ and positive integer $k$, there is a positive proportion of primes $\ell$ such that

$$
p\left(\frac{m^{k} \ell^{3} n+1}{24}\right) \equiv 0 \quad(\bmod m)
$$

holds for every nonnegative integer $n$ coprime to $\ell$. Weaver [30] gave an algorithm for finding the values of $\ell$ in (1.3) for primes $13 \leq m \leq 31$.

Ramanujan-type congruences of $p_{r}(n)$ have been extensively studied, see for example [2, 4, 11, 12, 14, 16, 19, 28]. Gandhi [1] derived the following congruences of $p_{r}(n)$ by applying the identities of Euler and Jacobi

$$
\begin{array}{rll}
p_{2}(5 n+3) & \equiv 0 \quad(\bmod 5), \\
p_{8}(11 n+4) & \equiv 0 \quad(\bmod 11) .
\end{array}
$$

With the aid of Sturm's theorem [27, Eichhorn and Ono 9] computed an upper bound $C\left(A, B, r, m^{k}\right)$ such that

$$
p_{r}(A n+B) \equiv 0 \quad\left(\bmod m^{k}\right)
$$

holds for all nonnegative integers $n$ if and only if it is true for $n \leq C\left(A, B, r, m^{k}\right)$. For example, to prove (1.4), it suffices to check that it holds for $n \leq 3$. In the same vain, one can prove (1.5) by verifying that it holds for $n \leq 11$. Treneer [28] extended (1.3) to weakly holomorphic modular forms and showed that for any prime $m \geq 5$ and positive integers $k$, there is a positive proportion of primes $\ell$ such that

$$
p_{r}\left(\frac{m^{k} \ell^{\mu_{r}} n+r}{24}\right) \equiv 0 \quad(\bmod m)
$$


for every nonnegative integer $n$ coprime to $\ell$, where $\mu_{r}$ equals to 1 if $r$ is even and 3 if $r$ is odd.

The aim of this paper is to study congruence properties of $p_{r}(n)$ modulo powers of primes. For example, we shall show that

$$
p_{r}\left(\frac{m^{k} \ell^{2 \mu K-1} n+r}{24}\right) \equiv 0 \quad\left(\bmod m^{k}\right)
$$

where $r$ is an odd integer, $\ell$ is a prime other than 2,3 and $m$, and $\mu$ is a positive integer, $K$ is a fixed positive integer, and $n$ is a positive integer coprime to $\ell$.

To derive congruences of $p_{r}(n)$, one may consider the congruence properties of the generating functions of $p_{r}(n)$. For the case of ordinary partitions, i.e., $r=1$, Chua [8] showed that

$$
\sum_{m n \equiv-1(\bmod 24)} p\left(\frac{m n+1}{24}\right) q^{n} \equiv \eta(24 z)^{\gamma_{m}} \phi_{m}(24 z) \quad(\bmod m)
$$

where $\eta(z)$ is Dedekind's eta function, $\gamma_{m}$ is an integer depending on $m$ and $\phi_{m}(z)$ is a holomorphic modular form. Ahlgren and Boylan [1] extended (1.7) to congruences modulo powers of primes, namely,

$$
F_{m, k}(z)=\sum_{m^{k} n \equiv-1(\bmod 24)} p\left(\frac{m^{k} n+1}{24}\right) q^{n} \equiv \eta(24 z)^{\gamma_{m, k}} \phi_{m, k}(24 z) \quad\left(\bmod m^{k}\right)
$$

where $\gamma_{m, k}$ is an integer and $\phi_{m, k}(z)$ is a holomorphic modular form.

In order to prove the existence of congruences of $p_{r}(n)$ modulo powers of primes, Brown and Li [7] introduced the generating function

$$
G_{m, k, r}(z) \equiv \sum_{\left(\frac{n}{m}\right)=-\left(\frac{-r}{m}\right)} p_{r}\left(\frac{n+r}{24}\right) q^{n}\left(\bmod m^{k}\right)
$$

and showed that $G_{m, k, r}(z)$ is a modular form of level $576 m^{3}$. Kilbourn [15] used the generating function

$$
H_{m, k, r}(z) \equiv \sum_{m n \equiv-r(\bmod 24)} p_{r}\left(\frac{m n+r}{24}\right) q^{n} \quad\left(\bmod m^{k}\right),
$$

and proved that $H_{m, k, r}(z)$ is a modular form of level $576 m$. However, due to the large dimensions of the spaces $M_{\lambda}\left(\Gamma_{0}\left(576 m^{3}\right)\right)$ and $M_{\lambda}\left(\Gamma_{0}(576 m)\right)$, it does not seem to be a feasible task to compute explicit bases. In other words, to derive explicit congruence formulas of $p_{r}(n)$, it is desirable to find 
a generating function of $p_{r}(n)$ that can be expressed in terms of modular forms of a small level.

In this paper, we find the following extension of the generating function $F_{m, k}(z)$, namely,

$$
F_{m, k, r}(z)=\sum_{m^{k} n \equiv-r(\bmod 24)} p_{r}\left(\frac{m^{k} n+r}{24}\right) q^{n},
$$

where $q=e^{2 \pi i z}$. We show that $F_{m, k, r}(z)$ is congruent to a meromorphic function modulo $m^{k}$. More precisely, we find

$$
F_{m, k, r}(z) \equiv \eta(24 z)^{\gamma_{m, k, r}} \phi_{m, k, r}(24 z) \quad\left(\bmod m^{k}\right)
$$

where $\gamma_{m, k, r}$ is an integer and $\phi_{m, k, r}(z)$ is a holomorphic modular form in $M_{\lambda_{m, k, r}}\left(\mathrm{SL}_{2}(\mathbb{Z})\right)$. Noting that any element of $M_{\lambda_{m, k, r}}\left(\mathrm{SL}_{2}(\mathbb{Z})\right)$ can be expressed as a polynomial of the Eisenstein series $E_{4}(z)$ and $E_{6}(z)$. This enable us to derive explicit congruences of generating functions of $p_{r}(n)$ modulo $m^{k}$.

If $\phi_{m, k, r}(z)=0$, then (1.12) yields a Ramanujan-type congruence as follows

$$
p_{r}\left(\frac{m^{k} n+r}{24}\right) \equiv 0 \quad\left(\bmod m^{k}\right) .
$$

For example, it is easily checked that $\phi_{5,1,2}(z)=0$ and $\phi_{11,1,2}(z)=0$, hence Gandhi's congruences (1.4) and (1.5) are the consequences of (1.13). We also find

$$
\begin{aligned}
p_{2}\left(5^{2} n+23\right) & \equiv 0 \quad\left(\bmod 5^{2}\right), \\
p_{8}\left(11^{2} n+81\right) & \equiv 0 \quad\left(\bmod 11^{2}\right),
\end{aligned}
$$

since $\phi_{5,2,2}(z)=0$ and $\phi_{11,2,8}(z)=0$. For more congruences of form (1.13), see Table 5.2 .

On the other hand, if $\phi_{m, k, r}(z) \neq 0$ in (1.12), we may use Yang's method [31] to find congruences of form (1.6). For example, since $F_{5,2,3}(z)$ is congruent to a modular form in the invariant space $S_{21,48}$ of $T_{5^{2}}$ modulo $5^{2}$, we have

$$
p_{3}\left(\frac{5^{2} \cdot 13^{199} n+3}{24}\right) \equiv 0 \quad\left(\bmod 5^{2}\right) .
$$

\section{Preliminaries}

To make this paper self-contained, we recall some definitions and facts on modular forms. In particular, we shall use the $U$-operator, the $V$-operator, the Hecke operator and the twist operator on the modular forms. 
Let $k \in \frac{1}{2} \mathbb{Z}$ be an integer or a half-integer, $N$ be a positive integer (with $4 \mid N$ if $k \notin \mathbb{Z})$ and $\chi$ be a Nebentypus character. We use $M_{k}\left(\Gamma_{0}(N), \chi\right)$ to denote the space of holomorphic modular forms on $\Gamma_{0}(N)$ of weight $k$ and character $\chi$. The corresponding space of cusp forms is denoted by $S_{k}\left(\Gamma_{0}(N), \chi\right)$. If $\chi$ is the trivial character, we shall write $M_{k}\left(\Gamma_{0}(N)\right)$ and $S_{k}\left(\Gamma_{0}(N)\right)$ for $M_{k}\left(\Gamma_{0}(N), \chi\right)$ and $S_{k}\left(\Gamma_{0}(N), \chi\right)$. Moreover, we write $\mathrm{SL}_{2}(\mathbb{Z})$ for $\Gamma_{0}(1)$.

Let $f(z) \in M_{k}\left(\Gamma_{0}(N), \chi\right)$ with the following Fourier expansion at $\infty$

$$
f(z)=\sum_{n \geq 0} a(n) q^{n}
$$

where $q=e^{2 \pi i z}$. Let us recall some operators acting on $f(z)$.

Let

$$
\gamma=\left(\begin{array}{ll}
a & b \\
c & d
\end{array}\right)
$$

be a $2 \times 2$ real matrix with positive determinant. The $k$ slash operator $\left.\right|_{k}$ is defined by

$$
\left(\left.f\right|_{k} \gamma\right)(z)=(\operatorname{det} \gamma)^{k / 2}(c z+d)^{-k} f(\gamma z)
$$

where

$$
\gamma z=\frac{a z+b}{c z+d}
$$

In particular, let $\ell$ be an integer and

$$
\gamma_{\ell}=\left(\begin{array}{cc}
0 & -1 \\
\ell & 0
\end{array}\right)
$$

The Fricke involution $W_{\ell}$ is given by

$$
f\left|W_{\ell}=f\right|_{k} \gamma_{\ell}
$$

The $U$-operator $U_{\ell}$ and $V$-operator $V_{\ell}$ are defined by

$$
f(z) \mid U_{\ell}=\sum_{n \geq 0} a(\ell n) q^{n}
$$

and

$$
f(z) \mid V_{\ell}=\sum_{n \geq 0} a(n) q^{\ell n} .
$$

It is known that

$$
\left.f(z)\right|_{k} U_{\ell}=\left.\ell^{\frac{k}{2}-1} \sum_{\mu=0}^{\ell-1} f(z)\right|_{k}\left(\begin{array}{cc}
1 & \mu \\
0 & \ell
\end{array}\right) .
$$


Let $\psi$ be a Dirichlet character. The $\psi$-twist of $f(z)$ is defined by

$$
(f \otimes \psi)(z)=\sum_{n \geq 0} \psi(n) a(n) q^{n} .
$$

Let $\ell$ be a prime and $f(z) \in M_{\lambda+\frac{1}{2}}\left(\Gamma_{0}(N), \chi\right)$ be a modular form of halfintegral weight. The Hecke operator $T_{\ell^{2}}$ is defined by

$$
f(z) \mid T_{\ell^{2}}=\sum_{n \geq 0}\left(a\left(\ell^{2} n\right)+\chi(\ell)\left(\frac{(-1)^{\lambda} n}{\ell}\right) \ell^{\lambda-1} a(n)+\chi\left(\ell^{2}\right) \ell^{2 \lambda-1} a\left(\frac{n}{\ell^{2}}\right)\right) q^{n} .
$$

We will use the following level reduction properties of the operators $U_{\ell}$ and $F_{\ell}=U_{\ell}+\ell^{\frac{k}{2}-1} W_{\ell}$ (see [17, Lemma 1] and [8, Lemma 2.2]).

Lemma 2.1 Let $k \in \mathbb{Z}, N$ be a positive integer, $\chi$ be a character modulo $N$, and $f(z) \in M_{k}\left(\Gamma_{0}(N), \chi\right)$. Assume that $\ell$ is a prime factor of $N$ and $\chi$ is also a character modulo $\mathrm{N} / \ell$.

(1) If $\ell^{2} \mid N$, then $f \mid U_{\ell} \in M_{k}\left(\Gamma_{0}(N / \ell), \chi\right)$.

(2) If $N=\ell$ and $\chi$ is the trivial character, then $f \mid F_{\ell} \in M_{k}\left(\mathrm{SL}_{2}(\mathbb{Z})\right)$.

In the proof of congruence (1.12) on the generating function $F_{m, k, r}(z)$, we need the following relation

$$
\eta(\gamma z)=\epsilon_{a, b, c, d}(c z+d)^{\frac{1}{2}} \eta(z),
$$

where $\gamma=\left(\begin{array}{ll}a & b \\ c & d\end{array}\right) \in \mathrm{SL}_{2}(\mathbb{Z}), \epsilon_{a, b, c, d}$ is a 24 -th root of unity, and $\eta(z)$ is Dedekind's eta function as given by

$$
\eta(z)=q^{\frac{1}{24}} \prod_{n=1}^{\infty}\left(1-q^{n}\right) .
$$

As a special case, we have

$$
\eta(-1 / z)=\sqrt{z / i} \cdot \eta(z) .
$$

\section{Generating functions of $p_{r}(n)$ modulo $m^{k}$}

In this section, we derive the congruence of the generating function $F_{m, k, r}(z)$ defined by (1.11), namely,

$$
F_{m, k, r}(z)=\sum_{m^{k} n \equiv-r(\bmod 24)} p_{r}\left(\frac{m^{k} n+r}{24}\right) q^{n} .
$$


Theorem 3.1 Let $m \geq 5$ be a prime, and let $k$ and $r$ be positive integers. Then there exists a modular form $\phi_{m, k, r}(z) \in M_{\lambda_{m, k, r}}\left(\mathrm{SL}_{2}(\mathbb{Z})\right)$, such that

$$
F_{m, k, r}(z) \equiv \eta(24 z)^{\gamma_{m, k, r}} \phi_{m, k, r}(24 z) \quad\left(\bmod m^{k}\right)
$$

where

$$
\begin{aligned}
\lambda_{m, k, r} & = \begin{cases}\frac{m^{k}-m^{k-1}}{2} r-\frac{\gamma_{m, k, r}+r}{2}, & \text { if } k \text { is odd }, \\
\left(m^{k}-m^{k-1}\right) r-\frac{\gamma_{m, k, r}+r}{2}, & \text { if } k \text { is even, }\end{cases} \\
\gamma_{m, k, r} & =\frac{24 \beta_{m, k, r}-r}{m^{k}},
\end{aligned}
$$

and $\beta_{m, k, r}$ is the unique integer in the range $0 \leq \beta_{m, k, r}<m^{k}$ congruent to $r / 24$ modulo $m^{k}$.

The first step of the proof of Theorem 3.1 is to express $F_{m, k, r}(z)$ in terms of a modular form. Consider the $\eta$-quotient

$$
f_{m, k, r}(z)=\left(\frac{\eta\left(m^{k} z\right)^{m^{k}}}{\eta(z)}\right)^{r}
$$

which is a cusp form in $S_{\frac{\left(m^{k}-1\right) r}{2}}\left(\Gamma_{0}\left(m^{k}\right),\left(\frac{\dot{m}}{m}\right)^{k r}\right)$. The following lemma shows that $F_{m, k, r}(z)$ can be obtained from $f_{m, k, r}(z)$ by applying a $U$-operator and a $V$-operator.

Lemma 3.2 Let $m \geq 5$ be a prime, and let $k$ and $r$ be positive integers. Then we have

$$
F_{m, k, r}(z)=\frac{\left(f_{m, k, r}(z) \mid U_{m^{k}}\right) \mid V_{24}}{\eta(24 z)^{m^{k} r}} .
$$

Proof. Since

$$
\sum_{n=0}^{\infty} p_{r}(n) q^{n}=\prod_{n=1}^{\infty} \frac{1}{\left(1-q^{n}\right)^{r}}
$$

we find

$$
\begin{aligned}
f_{m, k, r}(z) & =q^{\frac{m^{2 k}-1}{24} r} \prod_{n=1}^{\infty} \frac{1}{\left(1-q^{n}\right)^{r}} \cdot \prod_{n=1}^{\infty}\left(1-q^{m^{k} n}\right)^{m^{k} r} \\
& =q^{\frac{m^{2 k}-1}{24} r} \sum_{n=0}^{\infty} p_{r}(n) q^{n} \cdot \prod_{n=1}^{\infty}\left(1-q^{m^{k} n}\right)^{m^{k} r}
\end{aligned}
$$

Applying the operator $U_{m^{k}}$, we obtain

$$
f_{m, k, r}(z) \mid U_{m^{k}}=\sum_{n=0}^{\infty} p_{r}\left(m^{k} n+\beta_{m, k, r}\right) q^{n+\frac{r\left(m^{2 k}-1\right)+24 \beta_{m, k, r}}{24 m^{k}}} \cdot \prod_{n=1}^{\infty}\left(1-q^{n}\right)^{m^{k} r},
$$


where $0 \leq \beta_{m, k, r} \leq m^{k}-1$ is determined by $24 \beta_{m, k, r} \equiv r\left(\bmod m^{k}\right)$. So we deduce that

$$
\sum_{n=0}^{\infty} p_{r}\left(m^{k} n+\beta_{m, k, r}\right) q^{n+\frac{r\left(m^{2 k}-1\right)+24 \beta_{m, k, r}}{24 m^{k}}}=\frac{f_{m, k, r}(z) \mid U_{m^{k}}}{\prod_{n=1}^{\infty}\left(1-q^{n}\right)^{m^{k} r}} .
$$

Applying the operator $V_{24}$, we get

$$
\sum_{n=0}^{\infty} p_{r}\left(m^{k} n+\beta_{m, k, r}\right) q^{24 n+\frac{24 \beta_{m, k, r}-r}{m^{k}}}=\frac{\left(f_{m, k, r}(z) \mid U_{m^{k}}\right) \mid V_{24}}{\eta(24 z)^{m^{k} r}} .
$$

Replacing $24 n+\frac{24 \beta_{m, k, r}-r}{m^{k}}$ by $n$ in (3.6), or equivalently,

$$
n \rightarrow \frac{n}{24}-\frac{24 \beta_{m, k, r}-r}{24 m^{k}}
$$

one sees that the sum on the left hand side can be written in the form of $F_{m, k, r}(z)$. This completes the proof.

The second step of the proof of Theorem 3.1 is to derive a congruence relation for $f_{m, k, r}(z) \mid U_{m^{k}}$ modulo $m^{k}$.

Theorem 3.3 Let $m \geq 5$ be a prime, and let $k$ and $r$ be positive integers. Then there exists a modular form $G_{m, k, r}(z) \in M_{w_{m, k, r}}\left(\mathrm{SL}_{2}(\mathbb{Z})\right)$ such that

$$
f_{m, k, r}(z) \mid U_{m^{k}} \equiv G_{m, k, r}(z) \quad\left(\bmod m^{k}\right)
$$

where

$$
w_{m, k, r}= \begin{cases}\frac{2 m^{k}-m^{k-1}-1}{2} r, & \text { if } k \text { is odd }, \\ \frac{3 m^{k}-2 m^{k-1}-1}{2} r, & \text { if } k \text { is even } .\end{cases}
$$

Proof. Let

$$
g_{m, k, r}(z)=\left(\frac{\eta(z)^{m}}{\eta(m z)}\right)^{c_{k} m^{k-1} r}
$$

where

$$
c_{k}= \begin{cases}1, & \text { if } k \text { is odd } \\ 2, & \text { if } k \text { is even }\end{cases}
$$

Since $g_{m, k, r}(z)$ is an $\eta$-quotient, using the modular transformation property due to Gordon, Hughes, and Newman [13, 18,20], see also, [22, Theorem 1.64], we deduce that

$$
g_{m, k, r}(z) \in M_{\frac{c_{k}\left(m^{k}-m^{k-1}\right) r}{2}}\left(\Gamma_{0}(m),\left(\frac{\dot{m}}{m}\right)^{k r}\right) .
$$


Moreover, since $1-q^{m n} \equiv\left(1-q^{n}\right)^{m}(\bmod m)$, we see that

$$
g_{m, k, r}(z) \equiv 1 \quad\left(\bmod m^{k}\right) .
$$

Since $f_{m, k, r}(z) \in S_{\frac{\left(m^{k}-1\right) r}{2}}\left(\Gamma_{0}\left(m^{k}\right),\left(\frac{\dot{m}}{m}\right)^{k r}\right)$, using Lemma 2.1 repeatedly, we obtain that

$$
f_{m, k, r}(z) \mid U_{m^{k-1}} \in S_{\frac{\left(m^{k}-1\right) r}{2}}\left(\Gamma_{0}(m),\left(\frac{\cdot}{m}\right)^{k r}\right) .
$$

Thus, $f_{m, k, r}(z) \mid U_{m^{k-1}} \cdot g_{m, k, r}(z)$ is a modular form on $\Gamma_{0}(m)$ of the trivial character and of weight

$$
w_{m, k, r}=\frac{c_{k}\left(m^{k}-m^{k-1}\right) r}{2}+\frac{\left(m^{k}-1\right) r}{2} .
$$

Invoking Lemma 2.1, we find that

$$
G_{m, k, r}(z)=\left(f_{m, k, r}(z) \mid U_{m^{k-1}} \cdot g_{m, k, r}(z)\right) \mid F_{m}
$$

is a modular form in $M_{w_{m, k, r}}\left(\mathrm{SL}_{2}(\mathbb{Z})\right)$.

To complete the proof of Theorem 3.3, it remains to show that

$$
\left(f_{m, k, r}(z) \mid U_{m^{k-1}} \cdot g_{m, k, r}(z)\right)\left|F_{m} \equiv f_{m, k, r}(z)\right| U_{m^{k}} \quad\left(\bmod m^{k}\right)
$$

where

$$
F_{m}=U_{m}+m^{\frac{w_{m, k, r}}{2}-1} W_{m}
$$

and the operator $W_{m}$ is given by (2.2). By congruence (3.7), we see that the left hand side of (3.9) equals

$$
f_{m, k, r}(z)\left|U_{m^{k}}+m^{\frac{w_{m, k, r}}{2}-1}\left(f_{m, k, r}(z) \mid U_{m^{k-1}} \cdot g_{m, k, r}(z)\right)\right| W_{m} \quad\left(\bmod m^{k}\right) .
$$

To prove (3.9), it suffices to show that

$$
m^{\frac{w_{m, k, r}}{2}-1}\left(f_{m, k, r}(z) \mid U_{m^{k-1}} \cdot g_{m, k, r}(z)\right) \mid W_{m} \equiv 0 \quad\left(\bmod m^{k}\right) .
$$

We only consider the case when $k$ is odd. The case when $k$ is even can be dealt with in the same manner. In light of the transformation formula (2.9) of the eta function, we find that

$$
\begin{aligned}
g_{m, k, r}(z) \mid W_{m} & =m^{\frac{\left(m^{k}-m^{k-1}\right) r}{4}}(m z)^{-\frac{\left(m^{k}-m^{k-1}\right) r}{2}} g_{m, k, r}\left(-\frac{1}{m z}\right) \\
& =m^{-\frac{\left(m^{k}-m^{k-1}\right) r}{4}} z^{-\frac{\left(m^{k}-m^{k-1}\right) r}{2}}\left(\frac{(\sqrt{m z / i} \eta(m z))^{m}}{\sqrt{z / i} \eta(z)}\right)^{m^{k-1} r}
\end{aligned}
$$




$$
=m^{\frac{(m+1) m^{k-1} r}{4}}(-i)^{\frac{(m-1) m^{k-1} r}{2}}\left(\frac{\eta(m z)^{m}}{\eta(z)}\right)^{m^{k-1} r} .
$$

Therefore, (3.10) can be deduced from the following congruence

$$
m^{\frac{\left(3 m^{k}-1\right) r}{4}-1}\left(f_{m, k, r}(z) \mid U_{m^{k-1}}\right) \mid W_{m} \equiv 0 \quad\left(\bmod m^{k}\right) .
$$

By the property of $U$-operator as in (2.5), we have

$$
\begin{aligned}
& m^{\frac{\left(3 m^{k}-1\right) r}{4}-1} f_{m, k, r}(z)\left|U_{m^{k-1}}\right| W_{m} \\
= & \left.m^{\frac{(k+2) m^{k} r-(r+4) k}{4}} \sum_{\mu=0}^{m^{k-1}-1} f_{m, k, r}(z)\right|_{\frac{\left(m^{k}-1\right) r}{2}}\left(\begin{array}{cc}
1 & \mu \\
0 & m^{k-1}
\end{array}\right) \mid W_{m} \\
= & \left.m^{\frac{(k+2) m^{k} r-(r+4) k}{4}} \sum_{\mu=0}^{m^{k-1}-1} f_{m, k, r}(z)\right|_{\frac{\left(m^{k}-1\right) r}{2}}\left(\begin{array}{cc}
\mu m & -1 \\
m^{k} & 0
\end{array}\right) .
\end{aligned}
$$

Using the transformation formula (2.9) of the eta function, (3.12) can be written as

$$
\begin{aligned}
& m^{\frac{m^{k} r}{2}-k} z^{-\frac{\left(m^{k}-1\right) r}{2}} \sum_{\mu=0}^{m^{k-1}-1}\left(\frac{\eta\left(m \mu-\frac{1}{z}\right)^{m^{k}}}{\eta\left(\frac{m \mu z-1}{m^{k} z}\right)}\right)^{r} \\
= & m^{\frac{m^{k} r}{2}-k} z^{\frac{r}{2}} \eta(z)^{m^{k} r} \sum_{\mu=0}^{m^{k-1}-1} \frac{\alpha_{\mu}}{\eta\left(\frac{m \mu z-1}{m^{k} z}\right)^{r}},
\end{aligned}
$$

where $\alpha_{\mu}$ is a certain 24-th root unity.

For $\mu \neq 0$, we write $\mu=m^{s} t$ where $m \nmid t$. For $\mu=0$, we set $s=k-1$ and $t=0$. In either case, there exist integers $b$ and $d$ such that $b t+d m^{k-s-1}=-1$. It follows that

$$
\left(\begin{array}{cc}
m \mu & -1 \\
m^{k} & 0
\end{array}\right)=\left(\begin{array}{cc}
t & d \\
m^{k-s-1} & -b
\end{array}\right)\left(\begin{array}{cc}
m^{s+1} & b \\
0 & m^{k-s-1}
\end{array}\right) .
$$

Applying the corresponding slash operator to $\eta(z)$, we obtain that

$$
\eta\left(\frac{m \mu z-1}{m^{k} z}\right)=\epsilon_{\mu} m^{\frac{s+1}{2}} z^{\frac{1}{2}} \eta\left(\frac{m^{s+1} z+b}{m^{k-s-1}}\right),
$$

where $\epsilon_{\mu}$ is a 24-th root of unity. Since the coefficients of the Fourier expansion of $\eta(z)$ at $\infty$ are integers and the coefficient of the term with the lowest degree is 1, the Fourier coefficients of each term in (3.13) are divisible by $m^{\frac{m^{k}-s-1}{2} r-k}$ in the ring $\mathbb{Z}\left[\zeta_{24}\right]$. Clearly, $0 \leq s \leq k-1$. Thus we have

$$
\frac{m^{k}-s-1}{2} r-k \geq \frac{m^{k}-k}{2} r-k \geq \frac{m^{k}-k}{2}-k \geq k
$$


for $m \geq 5$ and $k \geq 1$. Hence the Fourier coefficients of each term in (3.13) are divisible by $m^{k}$. So we arrive at (3.11). This completes the proof.

We are now in a position to finish the proof of Theorem 3.1.

Proof of Theorem 3.1. By Theorem 3.3, there exists a modular form $G_{m, k, r}(z) \in$ $M_{w_{m, k, r}}\left(\mathrm{SL}_{2}(\mathbb{Z})\right)$ such that

$$
f_{m, k, r}(z) \mid U_{m^{k}} \equiv G_{m, k, r}(z) \quad\left(\bmod m^{k}\right) .
$$

Let

$$
\phi_{m, k, r}(z)=\frac{G_{m, k, r}(z)}{\Delta(z)^{\frac{m^{k} r+\gamma_{m, k, r}}{24}}},
$$

where $\Delta(z)=\eta(z)^{24}$ is Ramanujan's $\Delta$-function. In the proof of Lemma 3.2 we have shown that

$$
f_{m, k, r}(z) \mid U_{m^{k}}=\sum_{n=0}^{\infty} p_{r}\left(m^{k} n+\beta_{m, k, r}\right) q^{n+\frac{r\left(m^{2 k}-1\right)+24 \beta_{m, k, r}}{24 m^{k}}} \cdot \prod_{n=1}^{\infty}\left(1-q^{n}\right)^{m^{k} r},
$$

which implies that the order of the Fourier expansion of $f_{m, k, r}(z) \mid U_{m^{k}}$ at $\infty$ is at least

$$
\frac{r\left(m^{2 k}-1\right)+24 \beta_{m, k, r}}{24 m^{k}}=\frac{m^{k} r+\gamma_{m, k, r}}{24} .
$$

Thus $\phi_{m, k, r}(z)$ is a modular form in $M_{\lambda_{m, k, r}}\left(\mathrm{SL}_{2}(\mathbb{Z})\right)$. Combining (3.14) and Lemma 3.2, we conclude that

$$
\begin{aligned}
F_{m, k, r}(z) & \equiv \frac{\left(\Delta(z)^{\frac{m^{k} r+\gamma_{m, k, r}}{24}} \phi_{m, k, r}(z)\right) \mid V_{24}}{\eta(24 z)^{m^{k} r}} \\
& =\eta(24 z)^{\gamma_{m, k, r}} \phi_{m, k, r}(24 z) \quad\left(\bmod m^{k}\right),
\end{aligned}
$$

as required.

\section{Congruences of $p_{r}(n)$ modulo $m^{k}$}

In this section, we apply Theorem 3.1 on the congruence relation for the generating function $F_{m, r, k}(z)$ and Yang's method [31] to derive two classes of congruences of $p_{r}(n)$ modulo $m^{k}$.

Let

$$
S_{\gamma, \lambda}=\left\{\eta(24 z)^{\gamma} \phi(24 z): \phi(z) \in M_{\lambda}\left(\mathrm{SL}_{2}(\mathbb{Z})\right)\right\} .
$$

Yang [31] showed that when $\gamma$ is an odd integer such that $0<\gamma<24$ and $\lambda$ is a nonnegative even integer, $S_{\gamma, \lambda}$ is an invariant subspace of $S_{\lambda+\gamma / 2}\left(\Gamma_{0}(576), \chi_{12}\right)$ under the action of the Hecke algebra. More precisely, for all primes $\ell \neq 2,3$ and all $f \in S_{\gamma, \lambda}$, we have $f \mid T_{\ell^{2}} \in S_{\gamma, \lambda}$. By the invariant property of $S_{\gamma, \lambda}$, we obtain two classes of congruences of $p_{r}(n)$ modulo $m^{k}$. 
Theorem 4.1 Let $m \geq 5$ be a prime, $k$ be a positive integer, $r$ be an odd positive integer less than $m^{k}$, and $\ell$ be a prime different from 2,3 and $m$. Then there exists an explicitly computable positive integer $K$ such that

$$
p_{r}\left(\frac{m^{k} \ell^{2 \mu K-1} n+r}{24}\right) \equiv 0 \quad\left(\bmod m^{k}\right)
$$

for all positive integers $\mu$ and all positive integers $n$ relatively prime to $\ell$. There is also a positive integer $M$ such that

$$
p_{r}\left(\frac{m^{k} \ell^{i} n+r}{24}\right) \equiv p_{r}\left(\frac{m^{k} \ell^{2 M+i} n+r}{24}\right) \quad\left(\bmod m^{k}\right)
$$

for all nonnegative integers $i$ and $n$.

Proof. According to congruence relation (3.1), the generating function $F_{m, k, r}(z)$ is congruent to a modular form in $S_{\gamma_{m, k, r}, \lambda_{m, k, r}}$, where $\lambda_{m, k, r}$ and $\gamma_{m, k, r}$ are integers as given in (3.2) and (3.3). Let $\left\{f_{1}(z), \ldots, f_{d}(z)\right\}$ be a $\mathbb{Z}$-basis of the space $S_{\gamma_{m, k, r}, \lambda_{m, k, r}} \cap \mathbb{Z}[[q]]$ and

$$
f_{i}(z)=\sum_{n \geq 0} a_{i}(n) q^{n}
$$

where $i=1, \ldots, d$ and $q=e^{2 \pi i z}$.

To prove (4.1), it suffices to show that there exists a positive integer $K$ such that

$$
a_{i}\left(\frac{m^{k} \ell^{2 \mu K-1} n+r}{24}\right) \equiv 0 \quad\left(\bmod m^{k}\right)
$$

for all $n$ coprime to $\ell$ and $i=1, \ldots, d$.

From the relation $\gamma_{m, k, r} m^{k}=24 \beta_{m, k, r}-r$, one sees that $\gamma_{m, k, r}$ and $r$ have the same parity. Since $r<m^{k}$ is odd, we have $0<\gamma_{m, k, r}<24$, and hence $S_{\gamma_{m, k, r}, \lambda_{m, k, r}}$ is invariant under the Hecke operator $T_{\ell^{2}}$. So there exists a $d \times d$ matrix $A$ such that

$$
\left(\begin{array}{c}
f_{1} \\
\vdots \\
f_{d}
\end{array}\right) \mid T_{\ell^{2}}=A\left(\begin{array}{c}
f_{1} \\
\vdots \\
f_{d}
\end{array}\right)
$$

Let

$$
X=\left(\begin{array}{cc}
A & I_{d} \\
-\ell^{\gamma_{m, k, r}+2 \lambda_{m, k, r}-2} I_{d} & 0
\end{array}\right) .
$$

Using the property of the basis $\left\{f_{1}(z), \ldots, f_{d}(z)\right\}$ under the action of the $U$-operator as given by Yang [31, Corollary 3.4], we obtain

$$
\left(\begin{array}{c}
f_{1} \\
\vdots \\
f_{d}
\end{array}\right)\left|U_{\ell^{2}}^{s}=A_{s}\left(\begin{array}{c}
f_{1} \\
\vdots \\
f_{d}
\end{array}\right)+B_{s}\left(\begin{array}{c}
g_{1} \\
\vdots \\
g_{d}
\end{array}\right)+C_{s}\left(\begin{array}{c}
f_{1} \\
\vdots \\
f_{d}
\end{array}\right)\right| V_{\ell^{2}}
$$


where $s$ is a positive integer, $g_{i}=f_{i} \otimes(\dot{\bar{\ell}})$, and $A_{s}, B_{s}$ and $C_{s}$ are $d \times d$ matrices given by

$$
\begin{aligned}
& \left(\begin{array}{ll}
A_{s} & A_{s-1}
\end{array}\right)=\left(\begin{array}{ll}
I_{d} & 0
\end{array}\right) X^{s}, \\
& B_{s}=-\ell^{\lambda_{m, k, r}+\left(\gamma_{m, k, r}-3\right) / 2}\left(\frac{(-1)^{\left(\gamma_{m, k, r}-1\right) / 2} 12}{\ell}\right) A_{s-1}, \\
& C_{s}=-\ell^{\gamma_{m, k, r}+2 \lambda_{m, k, r}-2} A_{s-1} .
\end{aligned}
$$

Since $\operatorname{gcd}(m, \ell)=1$, the matrix $X\left(\bmod m^{k}\right)$ is invertible in the $\operatorname{ring} \mathcal{M}$ consisting of $2 d \times 2 d$ matrices over $\mathbb{Z}_{m^{k}}$. By the finiteness of $\mathcal{M}$, we see that there exist integers $a>b$ such that $X^{a}$ and $X^{b}$ are linear dependent over $\mathbb{Z}_{m^{k}}$, i.e., there exists a constant $c \in \mathbb{Z}_{m^{k}}$ such that $X^{a} \equiv c X^{b}\left(\bmod m^{k}\right)$. Thus $X^{K} \equiv c I_{2 d}\left(\bmod m^{k}\right)$, where $K=a-b$. In view of the relation

$$
\left(\begin{array}{ll}
A_{\mu K-1} & A_{\mu K-2}
\end{array}\right) \equiv c^{\mu}\left(\begin{array}{ll}
I_{d} & 0
\end{array}\right) X^{-1}\left(\bmod m^{k}\right),
$$

we find that $A_{\mu K-1} \equiv 0\left(\bmod m^{k}\right)$. Hence, from (4.5) it follows that

$$
\left(\begin{array}{c}
f_{1} \\
\vdots \\
f_{d}
\end{array}\right)\left|U_{\ell^{2}}^{\mu K-1} \equiv B_{\mu K-1}\left(\begin{array}{c}
g_{1} \\
\vdots \\
g_{d}
\end{array}\right)+C_{\mu K-1}\left(\begin{array}{c}
f_{1} \\
\vdots \\
f_{d}
\end{array}\right)\right| V_{\ell^{2}} \quad\left(\bmod m^{k}\right) .
$$

Applying the $U$-operator $U_{\ell}$ to both sides and observing that

$$
g_{i}\left|U_{\ell}=f_{i} \otimes(\dot{\bar{\ell}})\right| U_{\ell}=0,
$$

the relation (4.5) leads to the following congruence

$$
\left(\begin{array}{c}
f_{1} \\
\vdots \\
f_{d}
\end{array}\right)\left|U_{\ell^{2}}^{\mu K-1} U_{\ell} \equiv C_{\mu K-1}\left(\begin{array}{c}
f_{1} \\
\vdots \\
f_{d}
\end{array}\right)\right| V_{\ell} \quad\left(\bmod m^{k}\right)
$$

which implies (4.3).

We now turn to the proof of congruence (4.2). By the finiteness of $\mathcal{M}$, we see that there exists a positive integer $M$ such that $X^{M} \equiv I_{2 d}\left(\bmod m^{k}\right)$. Thus matrix equation (4.6) reduces to the following congruence

$$
\left(\begin{array}{ll}
A_{M} & A_{M-1}
\end{array}\right) \equiv\left(\begin{array}{ll}
I_{d} & 0
\end{array}\right) \quad\left(\bmod m^{k}\right) .
$$

It follows that $A_{M} \equiv I_{d}\left(\bmod m^{k}\right)$ and $B_{M} \equiv C_{M} \equiv 0\left(\bmod m^{k}\right)$. Thus, relation (4.5) implies

$$
\left(\begin{array}{c}
f_{1} \\
\vdots \\
f_{d}
\end{array}\right) \mid U_{\ell^{2}}^{M} \equiv\left(\begin{array}{c}
f_{1} \\
\vdots \\
f_{d}
\end{array}\right) \quad\left(\bmod m^{k}\right)
$$


So the coefficient of $q^{n}$ is congruent to the coefficient of $q^{\ell^{2 M}} n_{\text {in }} f_{i}(z)$ modulo $m^{k}$ for all $i$ and $n$. Since $F_{m, k, r}(z)$ is a linear combination of $f_{i}(z)$ with integer coefficients, we obtain congruence (4.2). This completes the proof.

\section{Examples}

In this section, we present some consequences of Theorem 3.1 and Theorem 4.1. We first give some examples for the congruences of the generating function $F_{m, k, r}(z)$ of $p_{r}(n)$.

Example 5.1 By Theorem 3.1, we find

$$
F_{m, k, r}(z) \equiv \eta(24 z)^{\gamma_{m, k, r}} \phi_{m, k, r}(24 z) \quad\left(\bmod m^{k}\right),
$$

where $\gamma_{m, k, r}$ is an integer, $\phi_{m, k, r}(z)$ is a polynomial of $\Delta(z)$ and the Eisenstein series $E_{4}(z)$ and $E_{6}(z)$. Table 5.1 gives the list of explicit expressions of $\eta(z)^{\gamma_{m, 1, r}} \phi_{m, 1, r}(z)$ for $m \leq 19$ and $2 \leq r \leq 7$.

\begin{tabular}{rr|l}
\hline$r$ & $m$ & $\eta(z)^{\gamma_{m, 1, r}} \phi_{m, 1, r}(z)$ \\
\hline 2 & 5 & 0 \\
& 7 & $3 \eta(z)^{10}$ \\
11 & $2 \eta(z)^{2} E_{4}(z)^{2}$ \\
13 & $8 \eta(z)^{22}$ \\
17 & $5 \eta(z)^{14} E_{4}(z)^{2}$ \\
19 & $\eta(z)^{10}\left(14 E_{4}(z)^{3}+12 \Delta(z)\right)$ \\
\hline 3 & 5 & $4 \eta(z)^{9}$ \\
& 7 & $3 \eta(z)^{3} E_{6}(z)$ \\
11 & 0 \\
13 & $\eta(z)^{9}\left(4 E_{4}(z)^{3}+6 \Delta(z)\right)$ \\
17 & 0 \\
19 & $\eta(z)^{15}\left(2 E_{6}(z)^{3}+3 E_{6}(z) \Delta(z)\right)$ \\
\hline 4 & 5 & $4 \eta(z)^{4} E_{4}(z)$ \\
& 7 & 0 \\
11 & $\eta(z)^{4}\left(3 E_{4}(z)^{4}+8 E_{4}(z) \Delta(z)\right)$ \\
13 & $\eta(z)^{20}\left(7 E_{4}(z)^{3}+4 \Delta(z)\right)$ \\
17 & $\eta(z)^{4}\left(6 E_{4}(z)^{7}+11 E_{4}(z)^{4} \Delta(z)+4 E_{4}(z) \Delta(z)^{2}\right)$ \\
19 & $\eta(z)^{20}\left(16 E_{4}(z)^{6}+18 E_{4}(z)^{3} \Delta(z)+2 \Delta(z)^{2}\right)$ \\
\hline 5 & 5 & $\eta(z)^{-1} E_{4}(z)^{2}$ \\
7 & $\eta(z)^{13} E_{6}(z)$ \\
11 & 0 \\
13 & $\eta(z)^{7}\left(8 E_{4}(z)^{6}+11 E_{4}(z)^{3} \Delta(z)+5 \Delta(z)^{2}\right)$ \\
17 & $\eta(z)^{11}\left(16 E_{4}(z)^{8}+16 E_{4}(z)^{5} \Delta(z)+4 E_{4}(z)^{2} \Delta(z)^{2}\right)$ \\
19 & $\eta(z)\left(5 E_{6}(z)^{7}+15 E_{6}(z)^{5} \Delta(z)+16 E_{6}(z)^{3} \Delta(z)^{2}\right)$ \\
\hline & & 14 \\
&
\end{tabular}




\begin{tabular}{rr|l}
\hline 6 & 5 & 0 \\
7 & $\eta(z)^{6}\left(6 E_{4}(z)^{3}+6 \Delta(z)\right)$ \\
11 & $\eta(z)^{6}\left(10 E_{4}(z)^{6}+E_{4}(z)^{3} \Delta(z)\right)$ \\
13 & $\eta(z)^{18}\left(7 E_{4}(z)^{6}+8 E_{4}(z)^{3} \Delta(z)+6 \Delta(z)^{2}\right)$ \\
17 & $\eta(z)^{18}\left(3 E_{4}(z)^{9}+3 E_{4}(z)^{6} \Delta(z)+5 E_{4}(z)^{3} \Delta(z)^{2}\right)$ \\
& 19 & $\eta(z)^{6}\left(6 E_{4}(z)^{12}+E_{4}(z)^{9} \Delta(z)+14 \Delta(z)^{4}\right)$ \\
\hline 7 & 5 & 0 \\
7 & $\eta(z)^{-1} E_{6}(z)^{3}$ \\
11 & 0 \\
13 & $\eta(z)^{5}\left(10 E_{4}(z)^{9}+6 E_{4}(z)^{6} \Delta(z)+9 E_{4}(z)^{3} \Delta(z)^{2}+11 \Delta(z)^{3}\right)$ \\
17 & $\eta(z)\left(7 E_{4}(z)^{13}+2 E_{4}(z)^{10} \Delta(z)+E_{4}(z)^{7} \Delta(z)^{2}+3 E_{4}(z)^{4} \Delta(z)^{3}\right)$ \\
19 & 0
\end{tabular}

Table 5.1: Explicit congruences derived from Theorem 3.1.

Example 5.2 Let $0 \leq \beta<m^{k}$ be an integer with $\beta \equiv r / 24\left(\bmod m^{k}\right)$. If $\phi_{m, k, r}(z) \equiv 0\left(\bmod m^{k}\right)$, using Theorem 3.1 we obtain the following Ramanujantype congruences of multipartition functions

$$
p_{r}\left(m^{k} n+\beta\right) \equiv 0 \quad\left(\bmod m^{k}\right)
$$

The values of $m$ and $\beta$ for $r \leq 9$ and $k=1,2$ are given in Table 5.2.

\begin{tabular}{|l|l|l|}
\hline$r$ & $(m, \beta)$ & $\left(m^{2}, \beta\right)$ \\
\hline 1 & $(5,4),(7,5),(11,6)$ & $(25,24),(49,47),(121,116)$ \\
\hline 2 & $(5,3)$ & $(25,23)$ \\
\hline 3 & $(11,7),(17,15)$ & $(121,106)$ \\
\hline 4 & $(7,6)$ & $(49,41)$ \\
\hline 5 & $(11,8),(23,5)$ & $(121,96)$ \\
\hline 6 & $(5,4)$ & $(25,19)$ \\
\hline 7 & $(5,3),(11,9),(19,9)$ & $(25,18),(121,86)$ \\
\hline 8 & $(7,5),(11,4)$ & $(121,81)$ \\
\hline 9 & $(17,11),(19,17),(23,9)$ & \\
\hline
\end{tabular}

Table 5.2: Ramanujan-type congruences of multipartitions.

It can be seen that Table 5.2 contains the Ramanujan congruences (1.1) of $p(n)$ modulo 5, 7 and 11, as well as Gandhi's congruences (1.4) for $p_{2}(n)$ and (1.5) for $p_{8}(n)$.

The following examples demonstrate how to derive certain congruences of $p_{r}(n)$ with the aid of Theorem 4.1. 
Example 5.3 For the values of $\ell$ and $K_{\ell}$ as given in Table 5.3, we have

$$
p_{3}\left(\frac{7 \cdot \ell^{2 \mu K_{\ell}-1} n+3}{24}\right) \equiv 0 \quad(\bmod 7)
$$

for all positive integers $\mu$ and all positive integers $n$ not divisible by $\ell$.

\begin{tabular}{r|rrrrrrrrrrrrrr}
\hline$\ell$ & 5 & 11 & 13 & 17 & 19 & 23 & 29 & 31 & 37 & 41 & 43 & 47 & 53 & 59 \\
\hline$a_{\ell}$ & 6 & 4 & 0 & 4 & 3 & 6 & 2 & 5 & 3 & 0 & 0 & 3 & 5 & 5 \\
$K_{\ell}$ & 6 & 7 & 2 & 6 & 8 & 7 & 7 & 8 & 3 & 2 & 2 & 8 & 3 & 8 \\
\hline
\end{tabular}

Table 5.3: Eigenvalues $a_{\ell}$ of $F_{7,1,3}(z)$ acted by $T_{\ell^{2}}$ and the corresponding $K_{\ell}$.

Proof. By Theorem 3.1 we find

$$
F_{7,1,3}(z) \equiv 3 \eta(24 z)^{3} E_{6}(24 z) \quad(\bmod 7) .
$$

Since $\eta(24 z)^{3} E_{6}(24 z)$ belongs to the 1-dimensional space $S_{3,6}$, for any prime $\ell \neq 2,3,7$, there exists an integer $a_{\ell}$ such that

$$
F_{7,1,3}(z) \mid T_{\ell^{2}} \equiv a_{\ell} F_{7,1,3}(z) \quad(\bmod 7)
$$

Inspecting the proof of Theorem 4.1, we obtain the corresponding orders $K_{\ell}$ for which congruence (5.2) holds.

Example 5.4 We have

$$
p_{3}\left(\frac{5^{2} \cdot 13^{199} n+3}{24}\right) \equiv 0 \quad\left(\bmod 5^{2}\right)
$$

for all integers $n$ coprime to 13 and

$$
p_{3}\left(\frac{5^{2} \cdot 13^{i} n+3}{24}\right) \equiv p_{3}\left(\frac{5^{2} \cdot 13^{200+i} n+3}{24}\right) \quad\left(\bmod 5^{2}\right)
$$

for all nonnegative integers $n$ and $i$.

Proof. By Theorem 3.1, $F_{5,2,3}(z)$ is congruent to a modular form in the space $S_{21,48}$ of dimension 5 . Setting

$$
f_{i}=\eta(24 z)^{21} E_{4}(24 z)^{3(5-i)} \Delta(24 z)^{i-1},
$$

for $1 \leq i \leq 5$. Clearly, $f_{1}, f_{2}, \ldots, f_{5}$ form a $\mathbb{Z}$-basis of $S_{21,48} \cap \mathbb{Z}[[q]]$. Let $A$ be the matrix of $T_{\ell^{2}}$ with respect to this basis. By computing the first five 
Fourier coefficients of $f_{i}$ and $f_{i} \mid T_{13^{2}}$ and equating the Fourier coefficients of both sides of (4.4), we find

$$
A \equiv\left(\begin{array}{ccccc}
17 & 21 & 18 & 3 & 3 \\
0 & 19 & 5 & 5 & 5 \\
0 & 0 & 22 & 4 & 19 \\
0 & 0 & 0 & 22 & 10 \\
0 & 0 & 0 & 0 & 12
\end{array}\right) \quad\left(\bmod 5^{2}\right)
$$

with the corresponding orders $K=M=100$. Setting $\mu=1$ in Theorem 4.1, we complete the proof.

Below are two more examples for $p_{3}(n)$ and $p_{5}(n)$ modulo $7^{2}$. The proofs are analogous to the proof of the above example, and hence are omitted.

Example 5.5 We have

$$
p_{3}\left(\frac{7^{2} \cdot 11^{2351} n+3}{24}\right) \equiv 0 \quad\left(\bmod 7^{2}\right)
$$

for all positive integers $n$ coprime to 7 and

$$
p_{3}\left(\frac{7^{2} \cdot 11^{i} n+3}{24}\right) \equiv p_{3}\left(\frac{7^{2} \cdot 11^{1176+i} n+3}{24}\right) \quad\left(\bmod 7^{2}\right)
$$

for all nonnegative integers $n$ and $i$.

Example 5.6 We have

$$
p_{5}\left(\frac{7^{2} \cdot 17^{195} n+5}{24}\right) \equiv 0 \quad\left(\bmod 7^{2}\right)
$$

for all positive integers $n$ coprime to 17 and

$$
p_{5}\left(\frac{7^{2} \cdot 17^{i} n+5}{24}\right) \equiv p_{5}\left(\frac{7^{2} \cdot 17^{588+i} n+5}{24}\right) \quad\left(\bmod 7^{2}\right)
$$

for all nonnegative integers $n$ and $i$.

Acknowledgments. This work was supported by the 973 Project, the PCSIRT Project of the Ministry of Education, and the National Science Foundation of China. 


\section{References}

[1] S. Ahlgren and M. Boylan, Arithmetic properties of the partition function, Invent. Math. 153 (2003) 487-502.

[2] G.E. Andrews, A survey of multipartitions: congruences and identities, In: Surveys in Number Theory, K. Alladi, ed., Developments in Mathematics, Vol. 17, Springer, 2008, pp. 1-19.

[3] A.O.L. Atkin, Proof of a conjecture of Ramanujan, Glasg. Math. J. 8 (1967) 14-32.

[4] A.O.L. Atkin, Ramanujan congruences for $p_{-k}(n)$, Canad. J. Math. 20 (1968) 67-78.

[5] A.O.L. Atkin and J.N. O'Brien, Some properties of $p(n)$ and $c(n)$ modulo powers of 13, Trans. Amer. Math. Soc. 126 (1967) 442-459.

[6] P. Bouwknegt, Multipartitions, generalized Durfee squares and affine Lie algebra characters, J. Austral. Math. Soc. 72 (2002) 395-408.

[7] J.L. Brown and Y.K. Li, Distribution of powers of the partition function modulo $\ell^{j}$, J. Number Theory 129 (2009) 2557-2568.

[8] K.S. Chua, Explicit congruences for the partition function modulo every prime, Arch. Math. 81 (2003) 11-21.

[9] D. Eichhorn and K. Ono, Congruences for partition functions, In: Analytic Number Theory, Vol. 1, Progr. Math. 138, Birkhauser, 1996, pp. 309-321.

[10] M. Fayers, Weights of multipartitions and representations of Ariki-Koike algebras, Adv. Math. 206 (2006) 112-144.

[11] J.M. Gandhi, Congruences for $p_{r}(n)$ and Ramanujan's $\tau$-function, Amer. Math. Monthly 70 (1963) 265-274.

[12] B. Gordon, Ramanujan congruence for $p_{-k}\left(\bmod 11^{r}\right)$, Glasgow Math. J. 24 (1983) 107-123.

[13] B. Gordon and K. Hughes, Multiplicative properties of eta-products II, Cont. Math. 143 (1993) 415-430.

[14] H. Gupta, Selected Topics in Number Theory, Abacus Press, Turnbridge Wells, 1980.

[15] T.P. Kilbourn, Congruence properties of Fourier coefficients of modular forms, Ph.D. Thesis, University of Illinois at Urbana-Champaign, 2007. 
[16] I. Kiming and J.B. Olsson, Congruences like Ramanujan's for powers of the partition function, Arch. Math. (Basel) 59 (4) (1992) 348-360.

[17] W. Li, Newforms and functional equations, Math. Ann. 212 (1975) 285315.

[18] M. Newman, Construction and application of a certain class of modular functions, Proc. London Math. Soc. 7 (3) (1956) 334-350.

[19] M. Newman, Some theorems about $p_{r}(n)$, Canad. J. Math. 9 (1957) 68-70.

[20] M. Newman, Construction and application of a certain class of modular functions (II), Proc. London Math. Soc. 9 (3) (1959) 373-387.

[21] K. Ono, Distribution of the partition function modulo $m$, Math. Ann. 151 (2000) 293-307.

[22] K. Ono, The Web of Modularity: Arithmetic of the Coefficients of Modular Forms and $q$-Series, CBMS 102, Amer. Math. Soc., Providence, 2004.

[23] S. Ramanujan, On certain arithmetical functions, Trans. Cambridge Philos. Soc. 22 (1916) 159-184.

[24] S. Ramanujan, Some properties of $p(n)$, the number of partitions of $n$, Proc. Cambridge Philos. Soc. 19 (1919) 207-210.

[25] S. Ramanujan, Congruence properties of partitions, Proc. London Math. Soc. III. Ser. 18 (1920) xix.

[26] S. Ramanujan, Congruence properties of partitions, Math. Z. 9 (1921) $147-153$.

[27] J. Sturm, On the congruence of modular forms, In: Lect. Notes Math. 1240 (1984), Springer Verlag, pp. 275-280.

[28] S. Treneer, Congruences for the coefficients of weakly holomorphic modular forms, Proc. London Math. Soc. 93 (2006) 304-324.

[29] G.N. Watson, Ramanujans vermutung über zerfällungsanzahlen, J. Reine Angew. Math. 179 (1938) 97-128.

[30] R. Weaver, New congruences for the partition function, Ramanujan J. 5 (2001) 53-63.

[31] Y. Yang, Congruences of the partition function, Internat. Math. Res. Notices 14 (2011) 3261-3288. 